H I G H L I G H TS

\section{To sleep, perchance to learn}

Sleep has fascinated humanity for centuries. Scientists have asked why we need to sleep, while mystics have thought that our dreams can tell us what the future holds. The mysteries of the dream world might still be out of reach, but researchers are coming closer to unravelling some of the functions of a good night's sleep.

It seems that while we are sleeping, our neurons might be busy remodelling the connections and circuits of the brain. Frank et al. have now shown that in young cats, plasticity in the visual cortex is enhanced by sleep at the critical period for visual system development.

During this critical period, the visual system of the cat is particularly sensitive to monocular deprivation. If visual input through one eye is blocked for a few hours, subsequent recordings from visual cortex show a reduction in responses to input through the deprived eye, and a decrease in the number of neurons showing binocular responsiveness.

Frank et al. investigated the effects of sleep on this form of plasticity. If cats were simply left in a dark room for six hours after the end of the period of monocular deprivation - and, cats being cats, they spent most of this time asleep - the changes in visual cortex responsiveness were much more marked than if the measurements were taken immediately after the deprivation. However, if the cats were put in a dark room but kept awake by a combination of movement of the cage floor and 'meowing' sounds being played whenever they started to drop off - the enhancement of plasticity was prevented. It seems that sleep, rather than time elapsed since the end

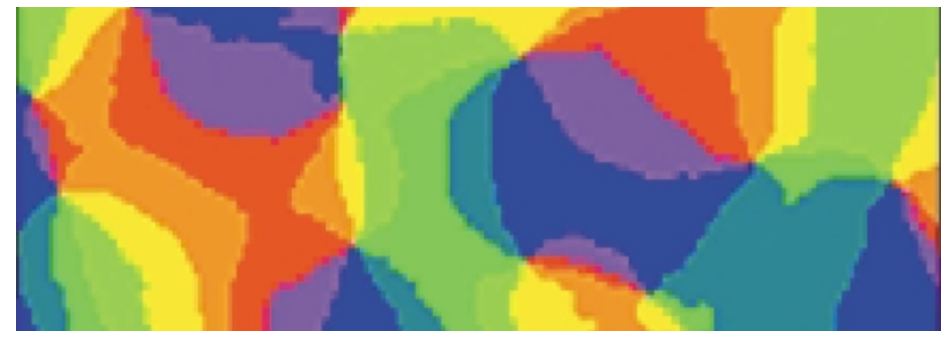

Orientation map of normal visual cortex. The colours represent the stimulus orientations to which neurons respond. Following monocular deprivation, the map becomes less sharp. (○) 2000 Macmillam Magazines.) of the deprived period or the absence of light, was responsible for the increase in the effect. In fact, six hours of sleep was at least as effective at increasing the changes in cortical responsiveness as an additional six hours of monocular deprivation.

Optical imaging confirmed the ing to map cortical responses to oriented stimuli presented to either the deprived eye or the control eye. Once again, responses to stimuli presented to the deprived eye were weaker and less selective, with the cats in the group that had slept showing the greatest effect.

Although rapid eye movement (REM) sleep has long been thought to be important for neuronal development in the young brain, Frank et al. found that the degree of enhancement of plasticity in the cats correlated with the amount of non-REM sleep during the six hours after monocular deprivation. This might tie in with the sharp increase in nonREM sleep that occurs in cats at the beginning of the critical period.

So it seems that our suspicions that sleep is essential for consolidation of memories and development or remodelling of neural pathways might be correct. Our dreams might still be a mystery, but at least we are starting to understand why sleep is so essential.

Rachel Jones

\section{(4) References and links} ORIGINAL RESEARCH PAPER Frank, M. G. et al. Sleep enhances plasticity in the developing visual cortex. Neuron 30, 275-287 (2001) FURTHER READING Sejnowski, T. J. \& Destexhe, A. Why do we sleep? Brain Res. $\mathbf{8 8 6}$ 208-223 (2000)

ENCYCLOPEDIA OF LIFE SCIENCES Sleep | Cortical plasticity: use-dependent remodelling WEB SITE Stryker lab results. Frank et al. used optical imag-

\section{DEVELOPMENT}

Calcium-dependent adhesion is necessary for the maintenance of prosomeres.

McCarthy, M. et al. Dev. Biol. 233, 80-94 (2001)

The authors examined the roles of calcium-dependent adhesion molecules in maintaining prosomeric boundaries in the mouse forebrain. Incorporation of cells into different parts of the forebrain depended on their cadherin expression profiles, but ectopic cadherin expression was not sufficient to cause a full switch in regional identity. Ectopic expression of R-cadherin in diencephalic precursors enabled their integration into the R-cadherin-expressing cortex, but did not activate cortical markers such as Emx-1 or BF-1.

\section{VESICLE PHYSIOLOGY}

Nucleoside diphosphate kinase, a source of GTP, is required for dynamin-dependent synaptic vesicle recycling.

Krishnan, K. S. et al. Neuron 30, 197-210 (2001)

Dynamin is a GTPase involved in endocytosis and required for vesicle recycling and downregulation of activated growth factor receptors. Krishnan et al. found that mutations in the Drosophila gene awd potentiate the temperature-sensitive block of dynamin in $s h i^{\text {ts }}$ mutant flies. As awd is an orthologue of the human tumour suppressor gene $n m 23$, these findings support the idea that such suppressors may endocytose activated growth factor receptors.

\section{EPILEPSY}

First genetic evidence of $\mathrm{GABA}_{A}$ receptor dysfunction in epilepsy: a mutation in the $\gamma 2$-subunit gene.

Baulac, S. et al. Nature Genet. 28, 46-48 (2001)

Mutant GABA $A_{A}$ receptor $\gamma 2$-subunit in childhood absence epilepsy and febrile seizures.

Wallace, R. H. et al. Nature Genet. 28, 49-52 (2001)

These studies show mutations in GABRG2, the $\gamma 2$-subunit of the $\mathrm{GABA}_{\mathrm{A}}$ receptor gene, in families with epilepsy. Baulac et al. found a mutation in a family with a disorder resembling generalized epilepsy with febrile seizures plus, whereas Wallace et al. found that the same gene was mutated in a family with childhood absence epilepsy and febrile seizures. These findings are the first proof that mutations in $\mathrm{GABA}_{\mathrm{A}}$ receptors are associated with epilepsy.

\section{GENE EXPRESSION}

Amygdala-enriched genes identified by microarray technology are restricted to specific amygdaloid subnuclei.

Zirlinger, M. et al. Proc. Natl Acad. Sci. USA 98, 5270-5275 (2001)

Zirlinger $e$ t al. used a combination of microarray analysis and in situ hybridization to investigate gene expression in the amygdala. Most of the genes that were enriched in the amygdala showed regional specificity, with boundaries of expression corresponding to the anatomical subnuclei. The analysis provides a set of molecular markers that will be useful for studying the roles of the amygdaloid subnuclei in emotional behaviours. 